Check for updates

Cite this: Mater. Adv., 2020, 1,3233

Received 19th July 2020,

Accepted 2nd October 2020

DOI: 10.1039/d0ma00522c

rsc.li/materials-advances

\title{
Binary treatment of PEDOT:PSS films with nitric acid and imidazolium-based ionic liquids to improve the thermoelectric properties $\dagger$
}

\author{
Temesgen Atnafu Yemata, ${ }^{\text {abc }}$ Yun Zheng, ${ }^{a}$ Aung Ko Ko Kyaw, (D) ad Xizu Wang, ${ }^{a}$ \\ Jing Song, ${ }^{a}$ Wee Shong $\mathrm{Chin}\left(\mathrm{DD}{ }^{* \mathrm{~b}}\right.$ and Jianwei $\mathrm{Xu}$ (D)*ab
}

\begin{abstract}
Poly(3,4-ethylenedioxythiophene):poly(styrenesulfonate) (PEDOT:PSS) is one of most prominent organic conductive polymer-based thermoelectric (TE) materials. Numerous combinations of doping and dedoping processes have been demonstrated to optimize the doping level of PEDOT:PSS films, and subsequently enhance electrical conductivity $(\sigma)$ and Seebeck coefficient $(S)$ of PEDOT:PSS films. This work established that binary post-treatment with nitric acid and imidazolium-based ionic liquids improved the $\sigma$ and $S$. The $\sigma$ of PEDOT:PSS film was significantly improved from 0.30 to $1260 \pm 61 \mathrm{~S} \mathrm{~cm}^{-1}$ and its corresponding $S$ was simultaneously increased from $16 \pm 1.2$ to $34.8 \pm 1.8 \mu \mathrm{V} \mathrm{K} \mathrm{K}^{-1}$, giving rise to a power factor of $152 \pm$ $11.2 \mu \mathrm{W} \mathrm{m} \mathrm{m}^{-1} \mathrm{~K}^{-2}$ at optimal conditions. The thermal conductivity $(\kappa)$ concurrently declined from 0.6 to $0.3 \mathrm{~W} \mathrm{~m}^{-1} \mathrm{~K}^{-1}$ for the untreated and treated PEDOT:PSS films, correspondingly, achieving a figure of merit $(Z T)$ value of $\sim 0.12$ at $300 \mathrm{~K}$. The TE performance enhancement of PEDOT:PSS films was largely attributable to the change in the doping level of PEDOT as evidenced by the overall increase in mobility and carrier concentration, and hence resulted in concurrent increase in the $\sigma$ and $S$, which were corroborated by different characterization methods. Studies on long-term stability of PEDOT:PSS films at $70{ }^{\circ} \mathrm{C}$ and $75 \% \mathrm{RH}$ for 20 days showed that more than $85 \% \sigma$ and $S$ was retained, showing potential applications for this binary post-treatment method.
\end{abstract}

\section{Introduction}

The emerging global need for a variety of efficient energyharvesting materials has been quickly increasing because of the significant industrialization and gradual depletion of fossil fuels. Recently, TE materials that convert thermal energy into electricity, and vice versa, have been paid a great deal of attention. ${ }^{1,2}$ TE modules play a vital role in sustainable development as they enable direct conversion between thermal energy and electrical energy by employing holes and electrons as energy transport carriers. Traditional TE materials are predominantly based on inorganic bulk material, for example,

\footnotetext{
${ }^{a}$ Institute of Materials Research and Engineering, Agency for Science, Technology and Research (A*STAR), 2 Fusionopolis Way, Singapore 138634, Republic of Singapore. E-mail: jw-xu@imre.a-star.edu.sg

${ }^{b}$ Department of Chemistry, National University of Singapore, 3 Science Drive 3, Singapore 117543, Republic of Singapore. E-mail: chmcws@nus.edu.sg ${ }^{c}$ Chemical Engineering Department, Bahirdar University, Bahirdar P.O. Box 26, Ethiopia

${ }^{d}$ Department of Electrical and Electronic Engineering, Southern University of Science and Technology, Shenzhen 518055, P. R. China

$\dagger$ Electronic supplementary information (ESI) available. See DOI: 10.1039/ d0ma00522c
}

PbTe-based compounds ${ }^{3,4}$ and $\mathrm{Bi}_{2} \mathrm{Te}_{3}$-based alloys. ${ }^{5,6}$ However, they have a few drawbacks such as some toxicity, high-cost, non-flexibility and relatively large thermal conductivity. ${ }^{7}$ In general, these inorganic materials are mainly applied with rigid bulk forms, making it difficult to be used on curved surfaces. Moreover, $\mathrm{PbTe}$-based materials perform efficiently only at high temperatures and are not appropriate for harvesting lowtemperature waste heat. Nevertheless, huge amounts of lowgrade waste heat, i.e. the temperature of less than $200{ }^{\circ} \mathrm{C},{ }^{8-10}$ is generated by various processes. Hence, conductive polymer TE materials with potential to provide high TE conversion efficiency at low temperature have attracted much attention in harvesting vast quantities of low-temperature waste heat. ${ }^{11}$ This is because conductive polymer TE materials possess intrinsically low $\kappa$, big $\sigma$, large-area fabrication, easy preparation, affordable cost as well as mass production. ${ }^{12}$

Recently, among the conductive polymer-based TE materials, PEDOT:PSS is in particular attractive due to its excellent thermal stability, good water processability, superior flexibility and high $\sigma$. However, a plethora of obstacles for this material have to be solved before extensive TE applications. For example, both the $\sigma$ and the $S$ of PEDOT:PSS need further enhancement to accomplish good TE performance. ${ }^{13}$ Several approaches have 
been reported such as post treatment with ethylene glycol and dimethyl sulfoxide, ${ }^{14}$ a sequential dipping process using polyethylenimine ethoxylated/ethylene glycol and $\mathrm{H}_{2} \mathrm{SO}_{4},{ }^{15}$ dimethyl sulfoxide-vapor annealing ${ }^{16}$ and PEDOT:PSS/MoS thin film with $4 \mathrm{wt} \% \mathrm{MoS}_{2}$ exfoliated in an $N, N$-dimethylformamide (DMF) solution. ${ }^{17}$ The magnitude of TE materials' conversion efficiency is defined as a unitless quantity, the figure of merit $(Z T), Z T=S^{2} \sigma T / \kappa$. In many cases, TE performance of a material can be assessed using power factor $\left(\mathrm{PF}=S^{2} \sigma\right)$ and it acts as an alternative to $Z T$ when the TE material has a very low $k$. Both high PF and a low $k$ are necessary to obtain a big $Z T$ value, but the conflicting relationship between the $\sigma$ and $S$ limits the further improvement of ZT. As a whole, high charge carrier concentration $(n)$ and/or high carrier mobility $(\mu)$ enable to achieve a high $\sigma$ value. However, a high $n$ value tends to decrease the $S .^{18-20}$ It has been demonstrated that the $\sigma$ value of PEDOT:PSS films could be greatly enhanced through a variety of post-treatment approaches. ${ }^{8,21-30}$ These post-treatment approaches increase the amount of $n$ and bipolarons which are the predominant charge carriers, but a large degree of the doping level usually leads to reduction in the $S$ on account of the presence of additional charge carriers. ${ }^{31}$ Therefore, apposite approaches that are able to improve the $S$ are strongly required. Furthermore, earlier investigation established that the PF could be enhanced by modulating the oxidation level via a chemical post-treatment means, ${ }^{32,33}$ achieving the optimization of TE properties via the modulation of the $n$ value. For example, Lee et al. studied a multiple-step post-treatment method by employing ultrafiltration and dedoping PEDOT:PSS with hydrazine $\left(\mathrm{NH}_{2} \mathrm{NH}_{2}\right)$, and eventually a maximum PF of $115.5 \mu \mathrm{W} \mathrm{m}{ }^{-1} \mathrm{~K}^{-2}$ was achieved. ${ }^{34}$ Also, Park's group described that PEDOT:PSS film underwent treatment with a mixture of dimethyl sulfoxide and $\mathrm{NH}_{2} \mathrm{NH}_{2}$ to afford a $\mathrm{PF}$ of $112 \mu \mathrm{W} \mathrm{m} \mathrm{m}^{-1} \mathrm{~K}^{-2} \cdot{ }^{31}$ Fan et al. post-treated the PEDOT:PSS films with $\mathrm{H}_{2} \mathrm{SO}_{4}$ and $\mathrm{NaOH}$, resulting in an enhanced $\mathrm{PF}$ of $334 \mu \mathrm{W} \mathrm{m}{ }^{-1} \mathrm{~K}^{-2}$ at ambient temperature. ${ }^{35}$ In their recent work, they used ionic liquid to treat PEDOT:PSS films, and obtained an extremely high PF of $754 \mu \mathrm{W} \mathrm{m}{ }^{-1} \mathrm{~K}^{-2},{ }^{36}$ being much greater than that obtained by Saxena et al. using a same ionic liquid $\left(\mathrm{PF}=170 \mu \mathrm{W} \mathrm{m}{ }^{-1} \mathrm{~K}^{-2}\right) .{ }^{37}$ Recently, we reported a series of work on post-treatment of PEDOT:PSS film using a combination of chemicals including a variety of reagents such as formamide, ${ }^{8}$ formic acid, ${ }^{38}$ trifluoroacetic acid, ${ }^{19}$ trifluoromethanesulfonic acid, ${ }^{24}$ hydrazine, ${ }^{38}$ formaldehyde sulfoxylate $^{39}$ and ionic liquid. ${ }^{40}$ For example, PEDOT:PSS films were post-treated with formamide and ionic liquids, and it was found that treatment only marginally had effect on the $\sigma$ but remarkably increased the $S$, achieving an overall PF up to $\sim 239.2 \mu \mathrm{W} \mathrm{m}{ }^{-1} \mathrm{~K}^{-2} \cdot{ }^{40}$

In addition to having outstanding TE performance, excellent long-term environmental stability is necessary for PEDOT:PSS film to be applied for useful TE modules, ${ }^{41-45}$ but limited studies have been reported. In this paper, we reported binary post-treatment with nitric acid $\left(\mathrm{HNO}_{3}\right)$ and ionic liquid to improve the $\sigma$ and $S$ values of PEDOT-PSS films as well as its environmental stability. Additionally, we chose one type of ionic liquid 1-butyl-3-methylimidazolium (bmim) with two different anions (trifluoromethanesulfonate (OTf) and tetrafluoroborate
$\left.\left(\mathrm{BF}_{4}\right)\right)$. Upon binary post-treatment of $\mathrm{HNO}_{3}$ and bmim-OTf in methanol, a highest PF of $152 \pm 11.2 \mu \mathrm{W} \mathrm{m}{ }^{-1} \mathrm{~K}^{-2}$ was achieved.

\section{Experimental}

\subsection{Materials}

PEDOT : PSS suspension with a weight ratio of PEDOT to PSS of $1: 2.5$ and the weight concentration of PEDOT:PSS of $1.3 \%$ (Clevios $\mathrm{PH}$ 1000) was purchased from Heraeus. $\mathrm{HNO}_{3}(70 \%)$ and ionic liquids ([bmim $][\mathrm{OTf}]$ ) and $\left[\mathrm{bmim}^{\mathrm{b}}\right]\left[\mathrm{BF}_{4}\right]$ were bought from Sigma-Aldrich. Other chemicals and reagents were used as received. Scheme 1 illustrates chemical structures of PEDOT:PSS and ionic liquids.

\subsection{Sample preparation}

Scheme 2 illustrates the overall procedures of film fabrication and post-treatment of PEDOT:PSS film. The pre-treatment of glass substrates and film preparation of the untreated PEDOT:PSS was conducted according to the reported procedures. ${ }^{29,39}$

2.2.1. $\mathrm{HNO}_{3}$ post-treatment. $\mathrm{HNO}_{3}(100 \mu \mathrm{l})$ was poured onto the PEDOT:PSS film at ambient temperature. After 10 minutes, the film was dried by passing $\mathrm{N}_{2}$ gas. ${ }^{46,47}$ Next, the films were immersed into the deionized $\mathrm{H}_{2} \mathrm{O}$ for three times to remove the excess $\mathrm{HNO}_{3}$ absorbed on the film surface. Finally, the resultant film was dried at $140{ }^{\circ} \mathrm{C}$ in an oven to take away the residual $\mathrm{H}_{2} \mathrm{O}$.

2.2.2. Ionic liquid post-treatment. Ionic liquids posttreatment was conducted at ambient temperature according to our reported method. ${ }^{40}$ The details of PEDOT:PSS film preparation and subsequent post-treatment with $\mathrm{HNO}_{3}$ and ionic liquids are summarized in Fig. S1 (ESI $\dagger$ ).

2.2.3. Instrumentation. The film thickness was measured on a KLA-Tencor P-10 surface profiler. The $\sigma$ of the PEDOT:PSS films was measured on a Laresta-GP MCP-T610 from Mitsubishi Chemical using the four-point probe method (Fig. S2, ESI $\dagger$ ). The $S$ of the PEDOT:PSS films were measured on a home-made setup according to our previous reported procedures (Fig. S3, ESI $\dagger$ ). ${ }^{39,45}$ Hall measurements including the $\mu$ and $n$ values were conducted on an Ecopia HMS-5000. Raman spectroscopy was obtained on a Renisha Raman system. UV-vis-NIR absorption spectra were recorded on a Shimadzu UV-vis spectrophotometer UV-3600.

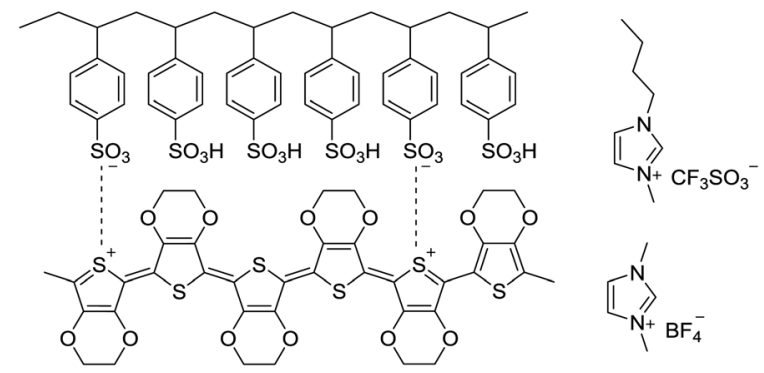

Scheme 1 Chemical structures of PEDOT:PSS, 1-ethyl-3-methylimidazolium (bmim) tetrafluoroborate and 1-butyl-3-methylimidazolium trifluoromethanesulfonate. 

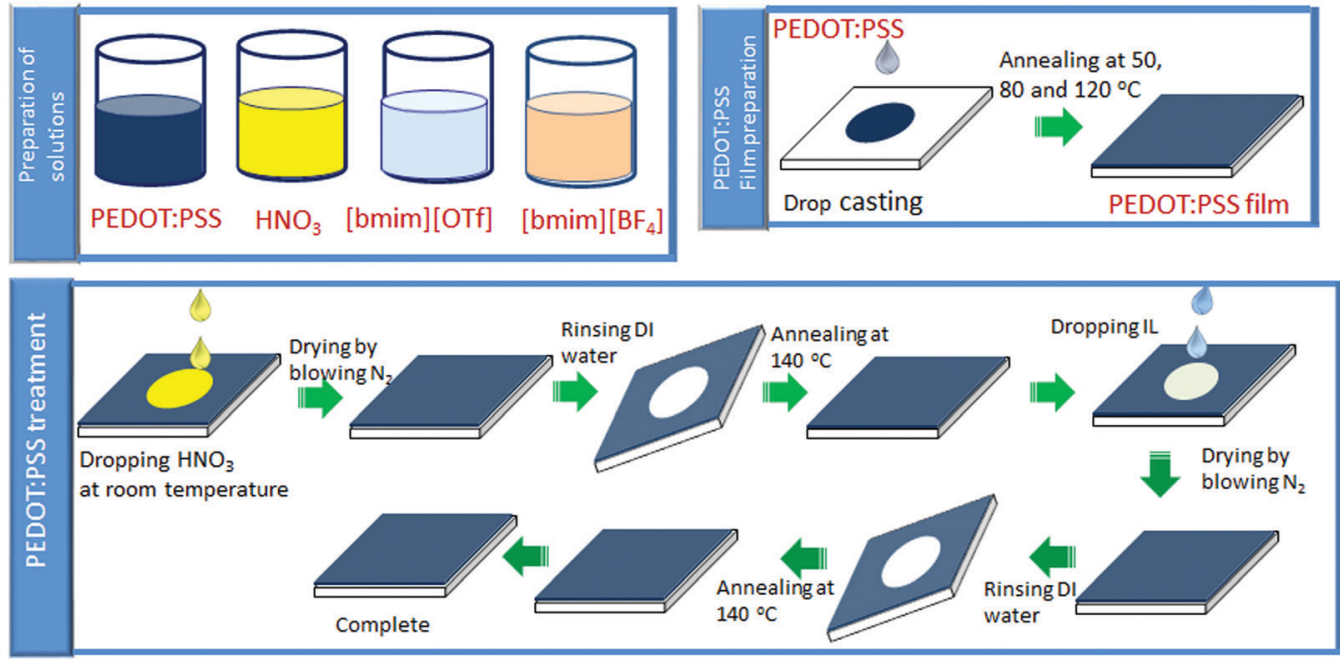

Scheme 2 Schematic illustration of fabrication of PEDOT:PSS films and its post-treatment.

X-ray photoelectron spectroscopy was performed on a Thermo Scientific to analyse the change in composition of PEDOT:PSS before and after treatment. The morphology and surface roughness were characterized by atomic force microscope (Bruker Dimension Icon). X-ray diffraction measurement was performed on a D8 Advance System, Bruker corporation to analyse the change in crystallinity of PEDOT:PSS films. The detailed characterizations of the PEDOT:PSS films are described in the ESI. $\dagger$

\section{Results and discussion}

\subsection{Thermoelectric properties}

TE properties of pristine and treated PEDOT:PSS films including the $\sigma$ and $S$ were studied. The untreated PEDOT:PSS film exhibited a small $\sigma$ value of $\sim 0.03 \pm 0.003 \mathrm{~S} \mathrm{~cm}^{-1}$ and an $S$ value of $\sim 17.5 \pm 1.6 \mu \mathrm{V} \mathrm{K}{ }^{-1}$. Post-treatment of PEDOT:PSS film with $\mathrm{HNO}_{3}$ for three times (designated as N-PEDOT:PSS, thereafter) resulted in an significant enhancement in the $\sigma$ from 0.03 to $3200 \pm 89 \mathrm{~S} \mathrm{~cm}^{-1}$, which approximately matches previously published data, ${ }^{43,48}$ and the $S$ value is merely marginally reduced from $17.5 \pm 1.6$ to $16.0 \pm 1.2 \mu \mathrm{V} \mathrm{K} \mathrm{K}^{-1}$. The N-PEDOT:PSS film was then subject to a similar treatment with ionic liquid to improve the $S$ value. The $\sigma, S$, and PF values of the untreated, N-PEDOT:PSS, [bmim][ $\left.\mathrm{BF}_{4}\right]$-N-PEDOT:PSS and [bmi$\mathrm{m}][$ OTf]-N-PEDOT:PSS films are summarized in Fig. 1 as well as in Table S1 (ESI $\dagger$ ). From the Fig. 1, it was found that the PF was increased to $76.0 \pm 5.3 \mu \mathrm{W} \mathrm{m}{ }^{-1} \mathrm{~K}^{-2}$ for the N-PEDOT:PSS film, mainly attributable to the remarkably enhanced $\sigma$ value. ${ }^{46,47}$ The N-PEDOT:PSS film treated with an ionic liquid [bmim][OTf] or $[\mathrm{bmim}]\left[\mathrm{BF}_{4}\right]$ caused the decline in $\sigma$ value from $3200 \pm 89$ to $1260 \pm 61$ and $1188 \pm 45 \mathrm{~S} \mathrm{~cm}^{-1}$, respectively. The $S$ of the N-PEDOT:PSS film was $16 \pm 1.2 \mu \mathrm{V} \mathrm{K}^{-1}$, which was faintly smaller than that of the untreated PEDOT:PSS film. However, the $S$ was greatly increased to $34.8 \pm 1.8$ and $33.9 \pm 1.9 \mu \mathrm{V} \mathrm{K}^{-1}$ for the N-PEDOT:PSS films treated with [bmim][OTf] and $\left[\mathrm{bmim}^{-}\left[\mathrm{BF}_{4}\right]\right.$, respectively. The N-PEDOT:PSS films dedoped with [bmim][OTf]

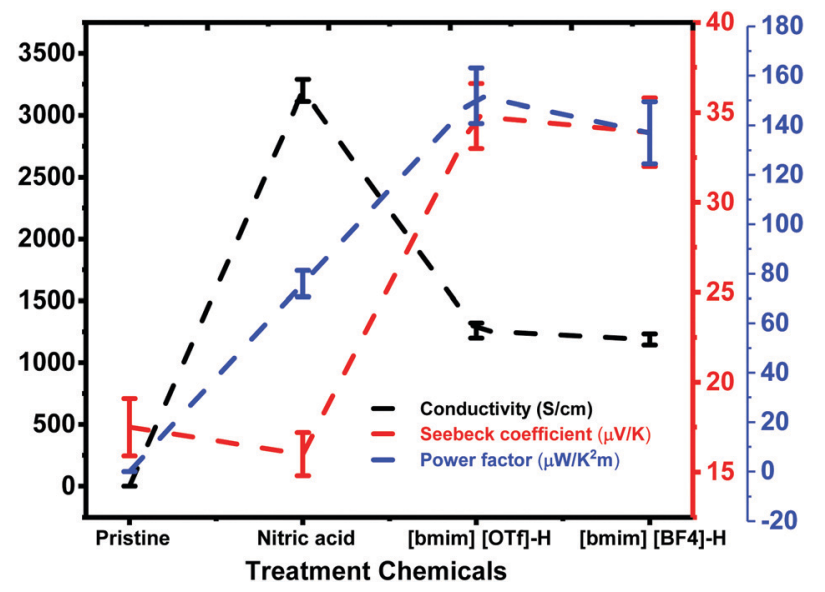

Fig. 1 The $\sigma, S$ and PF of the untreated, N-PEDOT:PSS, [bmim][OTf]-NPEDOT:PSS and $[\mathrm{bmim}]\left[\mathrm{BF}_{4}\right]-\mathrm{N}$-PEDOT:PSS films.

and $[\mathrm{bmim}]\left[\mathrm{BF}_{4}\right]$ exhibited both increase in $\sigma$ and $S$ in comparison with the untreated PEDOT:PSS film, thus resulting in an enhanced PF. The highest PFs of $\sim 152 \pm 11.2$ and $137 \pm$ $12.5 \mu \mathrm{W} \mathrm{m}{ }^{-1} \mathrm{~K}^{-2}$ were obtained from [bmim][OTf]-N-PEDOT:PSS and [bmim] $\left[\mathrm{BF}_{4}\right]-\mathrm{N}$-PEDOT:PSS, respectively. The two PF values obtained from both $\mathrm{HNO}_{3}$ and ionic liquid treatment are comparable favourably to these reported in literatures (Table S2, ESI $\dagger)^{34,49-51}$

\subsection{Characterization of TE properties of PEDOT:PSS films}

To understand the mechanism of the enhanced thermoelectric properties, the untreated, N-PEDOT:PSS, [bmim][OTf]-NPEDOT:PSS and [bmim] $\left[\mathrm{BF}_{4}\right]-\mathrm{N}$-PEDOT:PSS films were further characterized by UV-vis-NIR absorption spectroscopy, X-ray photoemission spectroscopy (XPS), Raman spectroscopy, X-ray diffraction (XRD) and atomic force microscopy (AFM).

The UV-vis absorption spectra and XPS spectra of the untreated, $\mathrm{HNO}_{3}$ and ionic liquid-treated PEDOT:PSS films were 

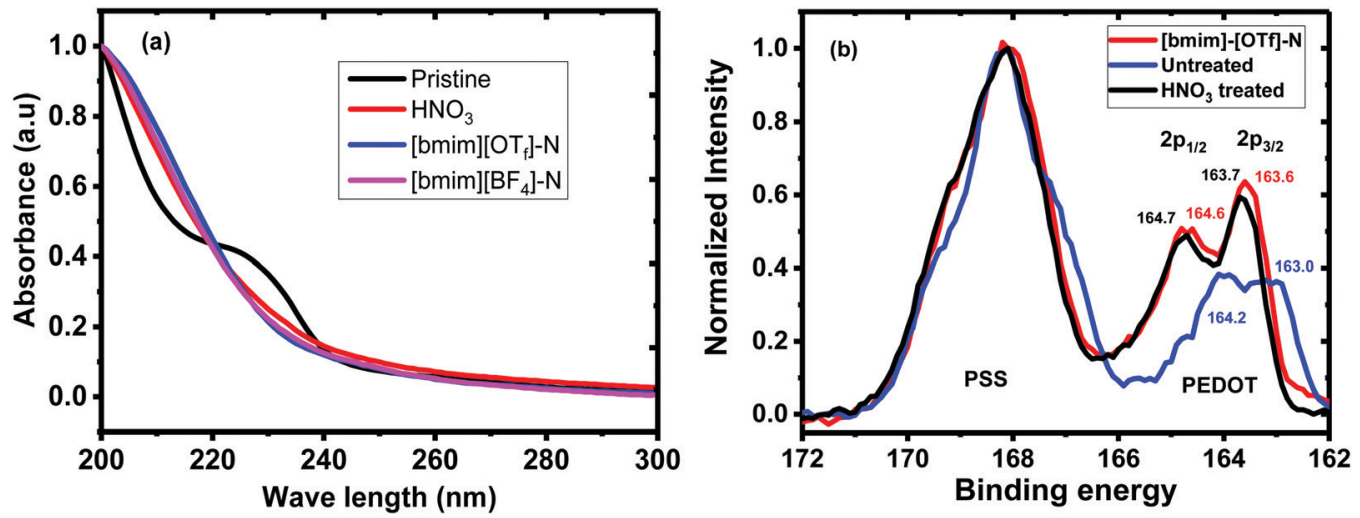

Fig. 2 (a) UV-vis absorbance spectra of untreated, N-PEDOT:PSS, [bmim][OTf]-N-PEDOT:PSS and [bmim][BF 4 ]-N-PEDOT:PSS films. (b) $S$ 2p XPS spectra of untreated, N-PEDOT:PSS and [bmim][OTf]-N-PEDOT:PSS films.

given in Fig. 2. The shoulder absorption peak centred at $225 \mathrm{~nm}$ corresponds to the phenyl groups of the PSS (Fig. 2a). The decline in the intensity of this absorption peak of the N-PEDOT:PSS, [bmim][OTf]-N-PEDOT:PSS and [bmim][BF $]$-N-PEDOT:PSS films showed the loss of PSS from the films which is in accordance with previous report. ${ }^{47}$ To further confirm the removal of PSS from the untreated PEDOT:PSS, N-PEDOT:PSS and [bmim][OTf]-NPEDOT:PSS films, the X-ray photoemission spectra of $\mathrm{S}_{2} \mathrm{p}$ core levels were characterized (Fig. 2b). The doublet peaks with binding energy in the range of 163 and $167 \mathrm{eV}$ are ascribed to the $\mathrm{S} 2 \mathrm{p}$ signal of the sulphur atoms in PEDOT, whereas the XPS peaks with binding energy from 167 and $170 \mathrm{eV}$ correspond to the $\mathrm{S} 2 \mathrm{p}$ signal of the sulphur atoms in $-\mathrm{SO}_{3} \mathrm{H}$ of PSS (Fig. S4, ESI $\dagger$ ). ${ }^{36,40,52}$ It was noteworthy that, compared with PSS, the relative intensity of $S$ 2p in PEDOT significantly enlarged because of the loss of PSS in the N-PEDOT:PSS and [bmim][OTf]-N-PEDOT:PSS films. ${ }^{53}$ Compared with $\mathrm{HNO}_{3}$ treated sample, the $\mathrm{S} 2 \mathrm{p}$ signals of the PEDOT film after dedoping with [bmim $][\mathrm{OTf}]$ slightly moved to the lower binding energy correspondingly, signifying the reduction in the doping level after treatment with [bmim][OTf] in conductive polymers unvaryingly causes a reduced $\sigma^{54}$ compared to films treated with $\mathrm{HNO}_{3}$. Since the $S$ increases at a lower doping level, the $\sigma$ has a tendency to decrease, and the PF of PEDOT:PSS film can attain its optimal value at a particular doping level.

UV-vis-NIR absorption spectra of the untreated, $\mathrm{N}$-PEDOT:PSS and N-[bmim][OTf]-PEDOT:PSS films and three intermediate structures that are composed of neutral (PEDOT), polaron $\left(\mathrm{PEDOT}^{+}{ }^{\bullet}\right)$ and bipolaron $\left(\mathrm{PEDOT}^{2+}\right)$, are illustrated in Fig. 3. These three PEDOT intermediate structures correspond to three absorption bands spanning from the visible to the near infrared (NIR) region: PEDOT $^{2+}$ shows a wide absorption band from the infrared region at about $1200 \mathrm{~nm}$ to larger NIR wavelengths. In contrast, $\mathrm{PEDOT}^{+}{ }^{\bullet}$ exhibits an absorption band centred at near $900 \mathrm{~nm}$ and the neutral PEDOT shows an absorption band centred at around $600 \mathrm{~nm} \cdot{ }^{55-58}$ The untreated film appears a wide absorption band starting from the visible region to the infrared region domain (Fig. 3). In contrast, the $\mathrm{HNO}_{3}$-treated PEDOT:PSS film appears a similar spectral profile to the untreated PEDOT:PSS film, but with stronger absorption than that in the untreated film, especially in NIR region, suggesting that the number of charge carriers (polaron and bipolaron) increases upon poste-treatment with $\mathrm{HNO}_{3}$, and

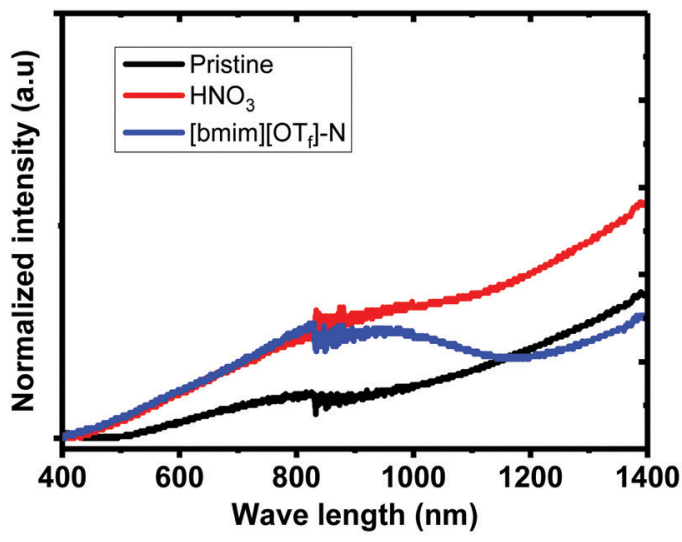

(a)

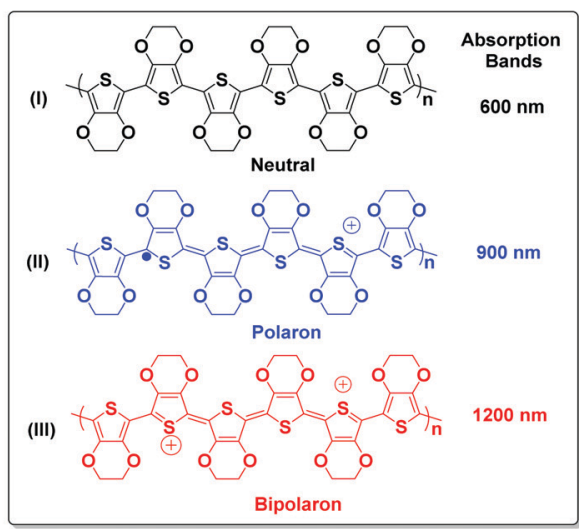

(b)

Fig. 3 UV-vis-NIR absorption spectra of the untreated, N-PEDOT:PSS and [bmim][OTf]-N-PEDOT:PSS films. Polymer PEDOT state: (I) neutral; (II) polaron and (III) bipolaron. 
polaron and bipolarons are primary charge carriers. This result in fact agrees with the observation showing the increase in the $\sigma$ and the reduction in the $S$. After further treatment of N-PEDOT:PSS film with [bmim][OTf], the broad IR absorption appears more pronounced at $\sim 900 \mathrm{~nm}$ associating with very obvious decrease in the absorption band centred at $c a .1200 \mathrm{~nm}$ that belongs to the absorption of bipolaron, revealing that PEDOT $^{2+}$ can be gradually dedoped with post-treatment with [bmim][OTf], and subsequently cause the substantial increase in the $S$ from around 16 to $\sim 35 \mu \mathrm{V} \mathrm{K}^{-1}$ (Fig. 1). Actually this observation is consistent with the downshift of the binding energy of $\mathrm{S}_{2} \mathrm{p}$ in comparison with $\mathrm{HNO}_{3}$-treated PEDOT:PSS film. In general, the binding energy of an atom decreases with increasing electron density about the atom. The less amount of bipolaron $\left(\mathrm{PEDOT}^{2+}\right.$ ) in ionic liquid-treated PEDOT:PSS film than in $\mathrm{HNO}_{3}$-treated PEDOT:PSS film means that the electron density of the overall sulphur atom increases after the posttreatment with ionic liquid, and therefore the binding energy of sulphur S 2p is downshifted.

To further investigate the doping mechanism, Raman spectra of the untreated, N-PEDOT:PSS, [bmim][OTf]-N-PEDOT:PSS and $[\mathrm{bmim}]\left[\mathrm{BF}_{4}\right]-\mathrm{N}-\mathrm{PEDOT}$ :PSS films were examined and are shown in Fig. 4. Three vibrational bands at around 987, 1130 and $1257 \mathrm{~cm}^{-1}$ were observed, which were attributed to the deformation of the $\mathrm{OCH}_{2}$ ring, the PSS part and the symmetric inter-aromatic ring vibrations of $\mathrm{C}_{\alpha}-\mathrm{C}_{\alpha^{\prime}}$ stretching, correspondingly. ${ }^{51,58-61}$ However, the low-intensity bands centred at $1130 \mathrm{~cm}^{-1}$ for $\mathrm{HNO}_{3}$-treated PEDOT:PSS film was weaker than that of untreated PEDOT:PSS film (Fig. S5, ESI $\dagger$ ), indicative of the loss of PSS after $\mathrm{HNO}_{3}$ treatment. Results extracted from UV-vis-NIR absorption and XPS spectra reveal that ionic liquid treatment dedopes the PEDOT to increase the polaron concentration, which is consistent with the increase in the $S$ and the drop in the $\sigma .^{62,63}$ Moreover, untreated PEDOT:PSS contains both benzoid and quinoid structures. The vibrational signal at $1421 \mathrm{~cm}^{-1}$ is ascribed to the stretching vibration of the $\mathrm{C}=\mathrm{C}$ double bond of thiophene group of the untreated PEDOT film. These vibrational peaks were moved to approximately $1411 \mathrm{~cm}^{-1}$ for the N-PEDOT:PSS indicating an

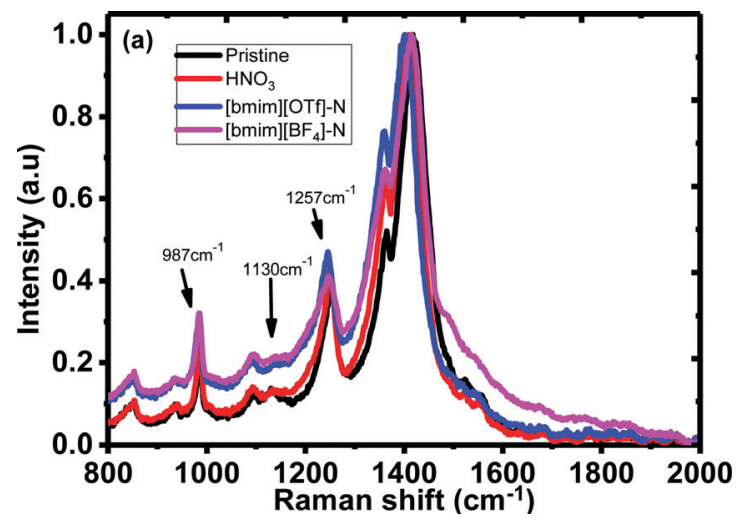

Fig. 4 Raman spectra of the untreated, N-PEDOT:PSS, [bmim][OTf]-NPEDOT:PSS and [bmim] $\left[\mathrm{BF}_{4}\right]-\mathrm{N}-\mathrm{PEDOT}$ :PSS films. alteration from a predominate coil alignment of benzoid configuration to a diverse linear-coil conformation of quinoid configuration in the PEDOT chain, ${ }^{59,64}$ leading to a quinoid dominant conformation. The PSS polymer chains are coupled with the PEDOT chains via attractive coulombic interactions to show core-shell structure thanks to repulsion among long PSS polymer chains. ${ }^{54,65-77}$ These treatments could deteriorate the ionic interaction between PSS and PEDOT. As a result, the phase separation between the PEDOT and PSS chains as well as the PEDOT chains with a linear conformation will be formed, thus eventually enabling $\sigma$ to become large with the assistance of the strong chain interactions. The stretching vibration of the $\mathrm{C}=\mathrm{C}$ double bond of thiophene group in N-PEDOT:PSS at $1411 \mathrm{~cm}^{-1}$ was red-shifted to $1402 \mathrm{~cm}^{-1}$ for [bmim][OTf]-N-PEDOT:PSS film but blue-shifted to $1416 \mathrm{~cm}^{-1}$ for [bmim][BF4]-NPEDOT:PSS film. Unlike the absorption spectra that give a clear evidence on dedoping of PEDOT by ionic liquids, the Raman spectra provide an inconsistent result, which is similar to a reported example. ${ }^{35}$ In our case, the exact reason of inconsistency on dedoping between Raman and absorption spectra might be due to the presence of different negative anions associated with [bmim].

The untreated, $\mathrm{HNO}_{3}$ and ionic liquid treated-PEDOT:PSS films was examined by AFM, and the height and phase AFM images are shown in Fig. 5. The roughness of height images of the untreated and $\mathrm{HNO}_{3}$-treated PEDOT:PSS films was similar to be 1.39 and $1.42 \mathrm{~nm}$, respectively, while the roughness of the height images for the corresponding PEDOT:PSS films was decreased to 0.98 and $0.80 \mathrm{~nm}$ after dedoping with [bmim][OTf] and $[\mathrm{bmim}]\left[\mathrm{BF}_{4}\right]$, respectively. The N-PEDOT:PSS and ionic liquid-treated N-PEDOT:PSS film surfaces exhibited very nonuniform, probably owing to an increase in the size of the PEDOT aggregates, making charge transport easier and hence increase in the $\sigma$ value. Similar to previous reports, the phase image of the untreated PEDOT:PSS film does not exhibit any apparent grains (Fig. 5), indicating that the PEDOT polymer chains are well inter-entangled with the PSS polymer chains and the film is mainly sheltered by the PSS-rich domains. The phase images of the N-PEDOT:PSS and ionic liquidtreated N-PEDOT:PSS were observed to be interconnected with large aggregates, inferring that the interconnection of the PEDOT-rich aggregates were originated from the significant phase separation between the PSS-rich shells and the PEDOTrich cores. ${ }^{67-69}$ Fig. S6 (ESI $\dagger$ ) presents the scanning electron microscope images of top-view and cross-section SEM images of the untreated, N-PEDOT:PSS, and ionic liquid treated N-PEDOT:PSS films. Contrary to the flat and smooth surface of the untreated PEDOT:PSS, it could be found that the crosssection SEM images of the ionic liquid-treated films demonstrated a layer-by-layer structure, which is in good agreement with the enhanced $\sigma .^{33,69}$

In an organic p-type semiconductor, the $\sigma$ value can be calculated according to the formula: $\sigma=e n \mu$, where $e$, and $\mu$ stand for the electronic charge and carrier mobility, respectively. In terms of the formula, it is clearly shown that the $\sigma$ value is proportional to both the $n$ and $\mu{ }^{63}$ The $n$ and $\mu$ values 

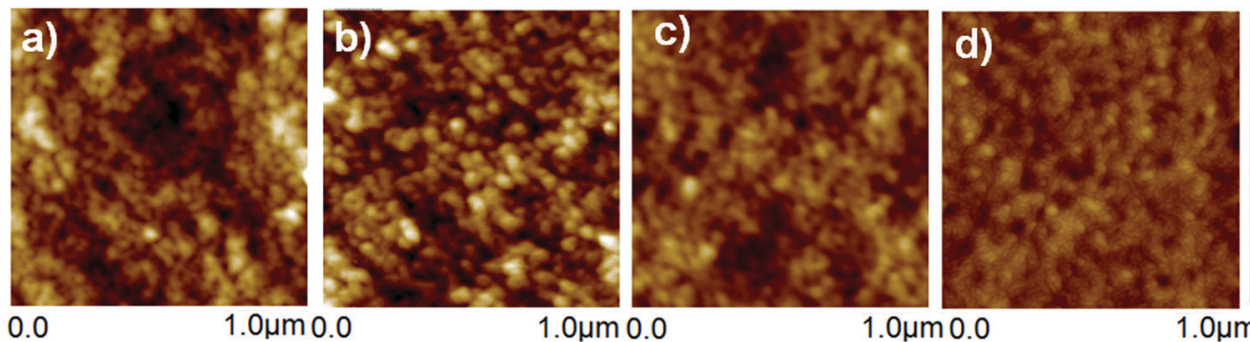

$5 \mathrm{~nm}$
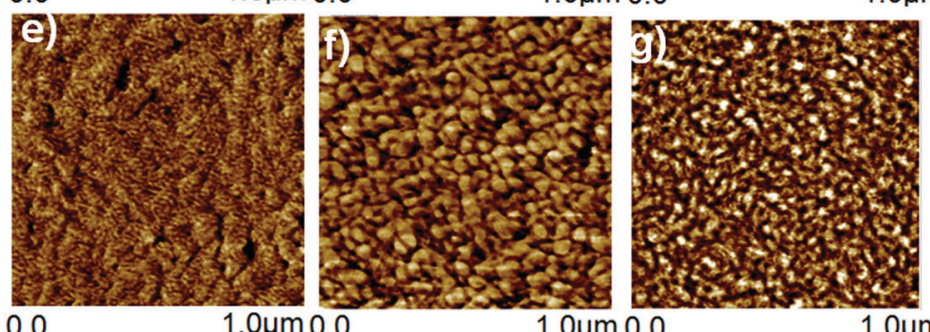

$1.0 \mu \mathrm{m} 0.0$

$1.0 \mu \mathrm{m} 0.0$

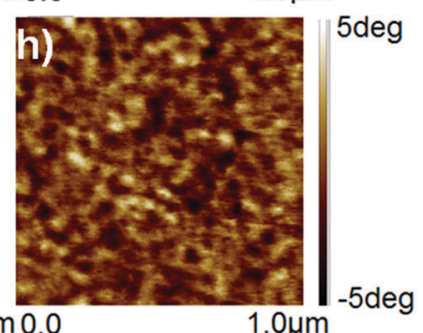

Fig. 5 The AFM images of PEDOT:PSS thin films. Height images (top): untreated (a) $\mathrm{HNO}_{3}$ (b) [bmim] [OTf]-N treated (c) [bmim] [BF $\left.\mathrm{B}_{4}\right]-\mathrm{N}$ treated (d) PEDOT:PSS films. Phase images (bottom): untreated (e), $\mathrm{HNO}_{3}$ (f), [bmim] [OTf]-N treated (g), [bmim] $\left[\mathrm{BF} \mathrm{f}_{4}\right]-\mathrm{N}$ treated (h) PEDOT:PSS films. The area scanned is $1 \times 1 \mu \mathrm{m}^{2}$ for each image.

of the untreated, N-PEDOT:PSS, [bmim][OTf]-N-PEDOT:PSS and [bmim $]\left[\mathrm{BF}_{4}\right]$-N-PEDOT:PSS films were measured, and are given in Table 1. It was found that the $\sigma$ of the ionic liquid-dedoped N-PEDOT:PSS films decreased by roughly $60 \%$ relative to that of the N-PEDOT:PSS film. The computed $\sigma$ of the untreated and treated-PEDOT:PSS films acquired from the product of the two-competing physical quantities $\mu$ and $n$ is the approximately same as the magnitude determined by the well-known four-point probe method (Table 1). As seen from Table 1, the largely reduced $\sigma$ of ionic liquid-dedoped PEDOT:PSS film was principally due to almost an order of magnitude reduction in the $n$. This perhaps stems from the interaction between the ionic liquid and the PSS components, hence suppressing the function of PSS as a carrier supplier of PEDOT. Post-treatment with ionic liquids with two different anions does not lead to the significant difference for the $\sigma, \mu$ and $n$ values, suggesting that the anions associated with the imidazolium cation play litter role in modulating the TE parameters, which in fact agrees well with the measured PF values (vide supra). Contrary to the big gap among the $n$ values with a difference of more four thousand times, the $\mu$ showed relatively less variation between $0.42-1.04\left(\mathrm{~cm}^{2} \mathrm{~V}^{-1} \mathrm{~s}^{-1}\right)$ with a difference of 2.5 times only for all samples.

XRD analysis was performed to examine the crystallinity of the PEDOT:PSS films (Fig. 6). For the untreated film, the patterns display two very weak characteristic signals at $2 \theta=6.5^{\circ}$ and $25.9^{\circ}$ which are assigned to the lattice $d$ spacing of 13.0 and $3.4 \AA$ calculated based on Bragg's law, $2 d \sin \theta=\lambda$. The $d$ spacing of $13.0 \AA\left(2 \theta \sim 6.5^{\circ}\right)$ corresponds to the lamella stacking distance $d(100)$ of the two distinct alternate ordering of PEDOT and PSS polymer chains. The $d$ spacing of $3.4 \AA$ spotted at $2 \theta=25.9^{\circ}$ corresponds to the inter-chain planar ring-stacking distance $d(010)$ of PEDOT chains. $^{39,70}$ The $\mathrm{N}$-PEDOT:PSS film shows an enormous change in intensity of $d(100)$ associating with obvious appearance of the second order diffraction of $d(100)$ at $2 \theta=13.0^{\circ}$, while a slight change in the lamella stacking distance of the two distinct alternate alignments of the PEDOT and PSS chain from 13.0 to $13.4 \AA$ was observed, and the $\pi-\pi$ stacking distance also was marginally increased from 3.4 to $3.5 \AA$ (Fig. 6). Upon $\mathrm{HNO}_{3}$ treatment, the increase in $\pi-\pi$ stacking distance indicates the transformation of the PEDOT chains from benzoid configuration to quinoid configuration, which results in the formation of a more planar structure. Moreover, the intensity of the diffraction peak $d(100)$ significantly increased after treatment with $\mathrm{HNO}_{3}$. A similar observation was also observed in $\mathrm{H}_{2} \mathrm{SO}_{4}$-treated PEDOT:PSS, suggesting that PEDOT:PSS favours a particular lamella stacking between the two distinct alternate orderings of PEDOT and PSS. ${ }^{71}$ The remarkable enhancement in its crystallinity was

Table 1 Experimentally measured $n, \mu$, and calculated $\sigma$ of the untreated, N-PEDOT:PSS, [bmim][OTf]-N-PEDOT:PSS and [bmim][BF 4 ]-N-PEDOT:PSS films

Post-treatment conditions

\begin{tabular}{|c|c|c|c|c|c|c|}
\hline Code & $\mathrm{HNO}_{3}$ & Ionic liquid & $\mu\left(\mathrm{cm}^{2} \mathrm{~V}^{-1} \mathrm{~s}^{-1}\right)$ & $n\left(\mathrm{~cm}^{-3}\right)$ & $\sigma^{a}\left(\mathrm{~S} \mathrm{~cm}^{-1}\right)$ & $\sigma^{b}\left(\mathrm{~S} \mathrm{~cm}^{-1}\right)$ \\
\hline ntreated & No & No & $0.42 \pm 0.03$ & $4.1 \pm 0.32 \times 10^{18}$ & $0.3 \pm 0.1$ & $0.29 \pm 0.11$ \\
\hline N-PEDOT:PSS & Yes & No & $1.04 \pm 0.10$ & $1.67 \pm 0.15 \times 10^{22}$ & $2778 \pm 196$ & $3200 \pm 89$ \\
\hline [bmim][OTf]-N-PEDOT:PSS & Yes & Yes & $0.65 \pm 0.05$ & $1.12 \pm 0.13 \times 10^{21}$ & $1164 \pm 112$ & $1260 \pm 61$ \\
\hline
\end{tabular}

${ }^{a}$ Calculated values in terms of $\sigma=e n \mu .{ }^{b}$ Obtained from the 4-point probe method. 


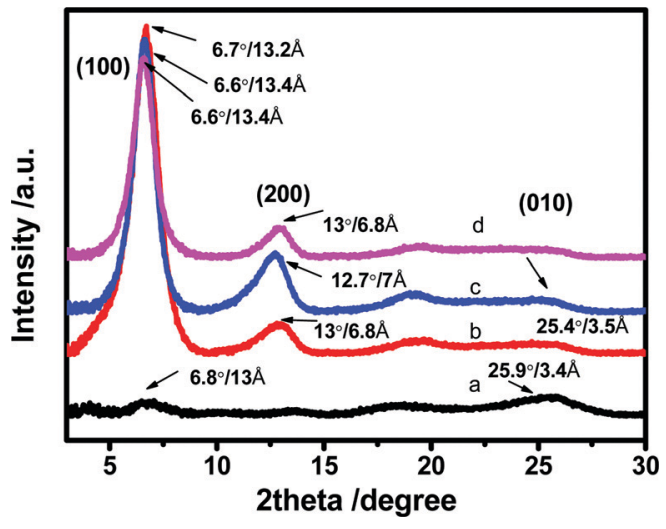

Fig. 6 X-ray diffraction (XRD) patterns of (a) untreated (b) $\mathrm{HNO}_{3}$ treated, (c) [bmim] [OTf]-N treated, and (d) [bmim] $\left[\mathrm{BF}_{4}\right]-\mathrm{N}$ treated PEDOT:PSS films.

also supported by the AFM images, being in good agreement with the reported results. ${ }^{46,47,71,72}$ By comparison, for the [bmim][OTf]-N-PEDOT:PSS and [bmim][BF 4 -N-PEDOT:PSS films, both diffractions of $d(100)$ at the low angle are slightly shifted to $\sim 6.6^{\circ}$, corresponding to the lattice $d$ spacing of $13.2 \AA$, and a fairly reduction in diffraction signal intensity is found, causing the reduction in crystallinity which is likely relevant to the decrease of the $\sigma$ after treatment with ionic liquids. The $d(100)$ diffraction peak intensity increased significantly, which was attributed to the increase in the number of ordered aggregates with interchain $\pi-\pi$ stacking between the PEDOT chains, as well as the increase in the crystallinity of the PEDOT:PSS film. Moreover, the addition of an ionic liquid selectively enhanced the $\pi-\pi$ coupling of the PEDOT:PSS chains, resulting in the improvement of crystallinity (i.e. reduction of disorder) and enhancing the charge carrier mobility. ${ }^{73,74}$ Consequently, the N-PEDOT:PSS, [bmim][OTf]-NPEDOT:PSS and/or [bmim $]\left[\mathrm{BF}_{4}\right]-\mathrm{N}-\mathrm{PEDOT}$ :PSS films exhibit improved interchain coupling of PEDOT:PSS chains with more densely packed PEDOT and lamella stacking between two types of assemblies, leading to enhanced $S$ of the PEDOT:PSS films through interface scattering. ${ }^{71,73,74}$ Interface scattering includes surface scattering and grain boundary scattering. The interface scattering mechanism of charge carriers transport in PEDOT:PSS film plays a role in the Seebeck coefficient and conductivity of PEDOT:PSS film. The post-treatment promotes the crystallinity of PEDOT and removes PSS at the same time, resulting in changes in the grain boundaries and grain size of PEDOT, and hence enhancing TE properties.

Table 2 presents density $(\rho)$ and thermal properties including thermal effusivity $(e)$, specific heat capacity $\left(C_{\mathrm{p}}\right)$ and cross-plane thermal conductivity $\left(\kappa_{\perp}\right)$ of the untreated and [bmim][OTf]-NPEDOT:PSS films. The cross-plane thermal conductivity $\left(\kappa_{\perp}\right)$ is given by the equation $\kappa=e^{2} / \rho C_{\mathrm{p}}$ where the $e$ is the thermal effusivity, which is measured by pulsed light heating thermoreflectance method at ambient temperature. ${ }^{21}$ This means renders the $e$ value of the film by determining the alternation in reflectance with pulsed light heating. Details of this method are elaborated in our earlier reports. ${ }^{29}$ Fig. S7 (ESI $\dagger$ ) displays the thermal reflectance signals of the untreated and
Table 2 Thermal properties and densities of the untreated and [bmim][OTf]N-PEDOT:PSS films

\begin{tabular}{lllll}
\hline Code & $\begin{array}{l}e\left(\mathrm{~J} \mathrm{~s}^{-0.5}\right. \\
\left.\mathrm{m}^{-2} \mathrm{~K}^{-1}\right)\end{array}$ & $\begin{array}{l}C_{\mathrm{p}} \\
\left(\mathrm{J} \mathrm{g}^{-1} \mathrm{~K}^{-1}\right) \rho\left(\mathrm{g} \mathrm{cm}^{-3}\right)\end{array}$ & $\begin{array}{l}\kappa_{\perp} \\
\left(\mathrm{W} \mathrm{m} \mathrm{m}^{-1} \mathrm{~K}^{-1}\right)\end{array}$ \\
\hline Untreated $^{\mathrm{a}}$ & 1196 & 1.50 & 1.60 & 0.60 \\
[bmim][OTf]-N- $^{\text {- }}$ & 856 & 1.40 & 1.59 & 0.30
\end{tabular}

PEDOT:PSS ${ }^{\mathrm{a}}$

[bmim][OTf]-N-PEDOT:PSS films. The thermal reflectance signals were monitored by the probe beam after the nanosecond-pulse heating. The untreated PEDOT:PSS film shows a quicker signal decay than the treated PEDOT:PSS film. This fast signal decay in the untreated film indicates that the cooling rate through the [bmim][OTf]-N-PEDOT:PSS film is slower than that through the untreated film and hence results in a faster thermal diffusion in the untreated PEDOT:PSS film. The effusivity values obtained from the curve fitting are summarized in Table 2. The $\kappa_{\perp}$ of [bmim][OTf]-N-PEDOT:PSS $\left(\sim 0.30 \mathrm{~W} \mathrm{~m}^{-1} \mathrm{~K}^{-1}\right)$ was dropped in comparison with the $\kappa_{\perp}$ of the untreated PEDOT:PSS film $\left(\sim 0.60 \mathrm{~W} \mathrm{~m}^{-1} \mathrm{~K}^{-1}\right)$ due to the selective loss of PSS, which is collectively confirmed by the UV-vis-NIR absorption, XPS and Raman spectra. The earlier investigation demonstrated that taking away the PSS from the PEDOT:PSS improved the $\sigma$, and only faintly lessened the $\kappa$ as well as decreased the $\rho$, but no close relationship between the $\kappa$ and $\sigma$ was found. ${ }^{34,76}$ The thermal anisotropy factor is equal to $\kappa_{\|} / \kappa_{\perp}$ where $\kappa_{\|}$and $\kappa_{\perp}$ stand for in-plane and cross-plane $\kappa$, respectively. The $\kappa_{\|} / \kappa_{\perp}$ value was found to be roughly ranging from $1.40 \pm 0.22^{57}$ to $3 .^{57,66}$ Therefore, the calculated $\kappa_{\|}$ranged from 0.84 to $1.8 \mathrm{~W} \mathrm{~m}^{-1} \mathrm{~K}^{-1}$ and from 0.42 to $0.9 \mathrm{~W} \mathrm{~m}^{-1} \mathrm{~K}^{-1}$ for the untreated and [bmim][OTf]-N-PEDOT:PSS films, respectively. Therefore, by utilizing the calculated $\kappa_{\|}$of $0.42-0.9 \mathrm{~W} \mathrm{~m}^{-1} \mathrm{~K}^{-1}$, the $Z T$ value at $300 \mathrm{~K}$ is estimated to be between from $\sim 0.12$ to $\sim 0.05$ for [bmim][OTf]-N-PEDOT:PSS film. While the $Z T$ value of untreated PEDOT:PSS film ranges from $\sim 3.6 \times 10^{-6}$ to $\sim 1.7 \times 10^{-6}$ at $300 \mathrm{~K}$ based on the estimated $\kappa_{\|}$of $0.84-1.8 \mathrm{~W} \mathrm{~m}^{-1} \mathrm{~K}^{-1}$ and PF of $0.01 \mu \mathrm{W} \mathrm{m} \mathrm{m}^{-1} \mathrm{~K}^{-2}$, confirming the effectiveness of our post-treatment method in improving the TE properties of PEDOT:PSS film.

\subsection{Stability study of PEDOT:PSS films}

Environmental stability of PEDOT:PSS films were investigated, and the untreated, N-PEDOT:PSS and [bmim][OTf]-NPEDOT:PSS films were exposed to air at $70{ }^{\circ} \mathrm{C}$ and a humidity of $75 \% \mathrm{RH}$ for 480 hours (20 days), and then the $\sigma, S$ and $\kappa$ values were regularly recorded throughout 20 days. As illustrated in Fig. 7, the $\sigma$ and $S$ of all PEDOT:PSS films progressively reduced with the increase in exposure time. After the PEDOT:PSS film was exposed to air at $70{ }^{\circ} \mathrm{C}$ and $75 \% \mathrm{RH}$ for 20 days, the $\sigma$ of the untreated, N-PEDOT:PSS and [bmim][OTf]N-PEDOT:PSS was reduced to 85,70 , and $30 \%$ of the original data, correspondingly, apparently implying that $\mathrm{HNO}_{3}$ treated PEDOT:PSS film has much better $\sigma$ stability than the untreated, and further treatment with [bmim][OTf] additionally improves its $\sigma$ stability and $85 \%$ of the original $\sigma$ is retained. Likewise, 


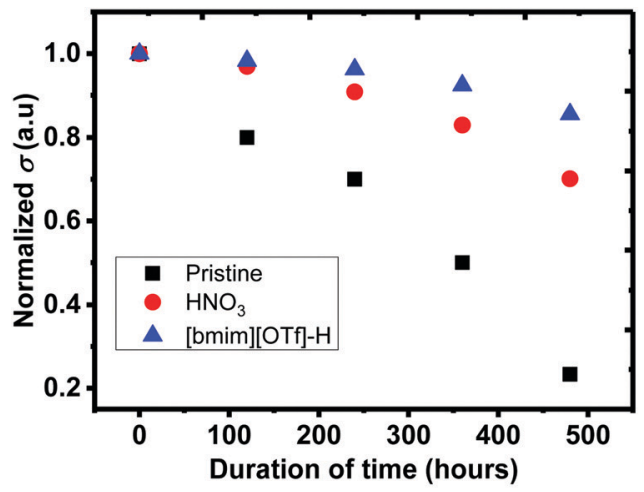

(a)

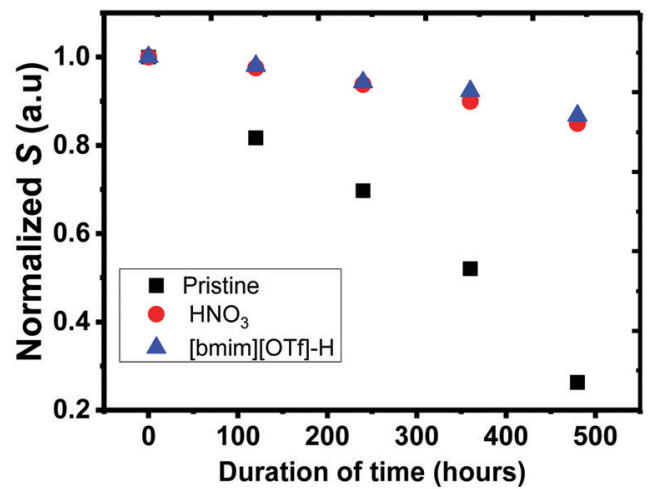

(b)

Fig. 7 The (a) $\sigma$ and (b) S of untreated, N-PEDOT:PSS and [bmim] [OTf]-N-PEDOT:PSS films over 480 hours at $70{ }^{\circ} \mathrm{C}$ and $75 \% \mathrm{RH}$. Vertical: $\sigma_{\mathrm{i}} / \sigma_{0}$ and $\mathrm{S}_{\mathrm{i}} / \mathrm{S}_{0}$ where $\left(\sigma_{i}\right.$ and $\left.S_{i}\right)$ and $\left(S_{0}\right.$ and $\left.\sigma_{0}\right)$ stand for $\sigma$ and $S$ of freshly-prepared ( 0 hour) and the time at which the $\sigma$ and $S$ were taken, respectively.

great improvement in $S$ was observed in $\mathrm{HNO}_{3}$ treated sample in comparison with the untreated sample, but the further treatment with ionic liquid did not lead to a notable change in the $S$ (Fig. 7b). It is noteworthy that, compared to the untreated PEDOT:PSS, remarkable improvement in both the $\sigma$ and $S$ of PEDOT:PSS film is primarily due to the great reduction of hygroscopic and strong acidic PSSH absorbing $\mathrm{H}_{2} \mathrm{O}$ easily. ${ }^{77,78}$ A less amount of the PSS in the treated PEDOT:PSS film leads to less $\mathrm{H}_{2} \mathrm{O}$ uptake and then the film becomes more intact even in wet conditions. ${ }^{68}$ Moreover, as demonstrated by XRD, the treated samples exhibited a much higher crystallinity than the untreated sample, hence enhancing the overall stability as reflected by the small reduction in the $\sigma$ and $S .^{37}$ On the other hand, strong electrostatic attraction between the negatively charged sulfonate anions and the positively charged bulky imidazolium cations may block the diffusion of $\mathrm{H}_{2} \mathrm{O}$ molecules from the film surface into the interior and consequently reduces the $\mathrm{H}_{2} \mathrm{O}$ uptake, partially contributing to the stability enhancement. In addition, neutralization happening between imidazolium derivatives with acidic PEDOT:PSS suspension additionally contributes to improvement in stability with a reasonable decline of the $\sigma$, which is consistent with reported result. ${ }^{44}$ It was also observed that weathering treatment also has an slight effect on the thermal properties (Table 2). Negligible change in $\kappa_{\perp}$ of the untreated PEDOT:PSS was observed, but led to around $13 \%$ increase after $\mathrm{HNO}_{3}$ and ionic liquid treatment. The environmental stability of both N-PEDOT:PSS and [bmim][OTf]-N-PEDOT:PSS films is better or comparable to that of these reported in literatures (Table S3, ESI $\dagger$ ). ${ }^{38-40,45,73}$

\section{Conclusions}

In this paper, we demonstrated post-treatments with $\mathrm{HNO}_{3}$ treatment considerably improved the $\sigma$ of the untreated PEDOT:PSS film from 0.03 to more than $3000 \mathrm{~S} \mathrm{~cm}^{-1}$, however resulting in a slight drop in the $S$. Further dedoping $\mathrm{HNO}_{3}$ treated film with ionic liquids increased $S$ by more than double from $16 \pm 1.2$ to $34.8 \pm 1.8 \mu \mathrm{V} \mathrm{K}^{-1}$ via adjusting the oxidation level of the PEDOT films. Binary treatment with $\mathrm{HNO}_{3}$ and ionic liquid substantially improved the $\sigma$ and $S$ values, leading to a PF value of $152 \pm 11.2 \mu \mathrm{W} \mathrm{m}{ }^{-1} \mathrm{~K}^{-2}$ at optimal conditions. The $\kappa$ was decreased from $\sim 0.6$ to $0.3 \mathrm{~W} \mathrm{~m}^{-1} \mathrm{~K}^{-1}$ for the untreated and the treated film, respectively. Therefore, the $Z T$ of $\sim 0.12$ was obtained at $300 \mathrm{~K}$. The long-term environmental stability study at $70{ }^{\circ} \mathrm{C}$ and $75 \% \mathrm{RH}$ for 20 days demonstrated that the PEDOT:PSS films treated with nitric acid and ionic liquid were stable and more than $85 \%$ of its $\sigma$ and $S$ were retained, showing potential in real applications such as thin-film TE generators.

\section{Conflicts of interest}

The authors declare no conflict of interest.

\section{Acknowledgements}

We are grateful to support from the A*STAR, Industry Alignment Funding, (Grant No. 1527200019, 1527200020 \& 1527200021). Yemata would like to specially thank the SINGA scholarship from the ${ }^{*}$ STAR Graduate Academy. Kyaw would like to acknowledge the support from Shenzhen Science, Technology and Innovation Commission (No. JCYJ20180305180645221) and High-level University Fund (G02236004).

\section{References}

1 S. B. Riffat and X. Ma, Appl. Therm. Eng., 2003, 23, 913-935.

2 L. E. Bell, Science, 2008, 321, 1457-1461.

3 Y. Pei, X. Shi, A. LaLonde, H. Wang, L. Chen and G. J. Snyder, Nature, 2011, 473, 66.

4 K. Biswas, J. He, I. D. Blum, C.-I. Wu, T. P. Hogan, D. N. Seidman, V. P. Dravid and M. G. Kanatzidis, Nature, 2012, 489, 414.

5 Y. Zheng, Q. Zhang, X. Su, H. Xie, S. Shu, T. Chen, G. Tan, Y. Yan, X. Tang and C. Uher, Adv. Energy Mater., 2015, 5, 1401391. 
6 B. Poudel, Q. Hao, Y. Ma, Y. Lan, A. Minnich, B. Yu, X. Yan, D. Wang, A. Muto and D. Vashaee, Science, 2008, 320, 634-638. 7 M. O'Neill and S. M. Kelly, Adv. Mater., 2011, 23, 566-584.

8 Q. Wei, M. Mukaida, K. Kirihara, Y. Naitoh and T. Ishida, Materials, 2015, 8, 732-750.

9 N. Toshima and S. Ichikawa, J. Electron. Mater., 2015, 44, 384-390.

10 D. Yoo, J. Kim, S. H. Lee, W. Cho, H. H. Choi, F. S. Kim and J. H. Kim, J. Mater. Chem. A, 2015, 3, 6526-6533.

11 N. Dubey and M. Leclerc, J. Polym. Sci., Part B: Polym. Phys., 2011, 49, 467-475.

12 Y. Du, S. Z. Shen, K. Cai and P. S. Casey, Prog. Polym. Sci., 2012, 37, 820-841.

13 N.-R. Shin, S.-H. Choi and J.-Y. Kim, Synth. Met., 2014, 192, 23-28.

14 J. Liu, Y. Jia, Q. Jiang, F. Jiang, C. Li, X. Wang, P. Liu, P. Liu, F. Hu, Y. Du and J. Xu, ACS Appl. Mater. Interfaces, 2018, 10, 44033-44040.

15 X. Li, C. Liu, W. Zhou, X. Duan, Y. Du, J. Xu, C. Li, J. Liu, Y. Jia, P. Liu, Q. Jiang, C. Luo, C. Liu and F. Jiang, ACS Appl. Mater. Interfaces, 2019, 11, 8138-8147.

16 X. Wang, P. Liu, Q. Jiang, W. Zhou, J. Xu, J. Liu, Y. Jia, X. Duan, Y. Liu, Y. Du and F. Jiang, ACS Appl. Mater. Interfaces, 2018, 11, 2408-2417.

17 F. Jiang, J. Xiong, W. Zhou, C. Liu, L. Wang, F. Zhao, H. Liu and J. Xu, J. Mater. Chem. A, 2016, 4, 5265-5273.

18 Q. Jiang, Q. Liu, H. Song, H. Shi, Y. Yao, J. Xu, G. Zhang and B. Lu, J. Mater. Sci.: Mater. Electron., 2013, 24, 4240-4246.

19 J. Kim, J. G. Jang, J.-I. Hong, S. H. Kim and J. Kwak, J. Mater. Sci.: Mater. Electron., 2016, 27, 6122-6127.

20 L. M. Cowen, J. Atoyo, M. Carnie, D. Baran and B. C. R. Schroeder, ECS J. Solid State Sci. Technol., 2017, 6, N3080-N3088.

21 A. K. K. Kyaw, G. D. H. Wong, T. A. Yemata and J. Xu, Org. Electron., 2019, 69, 7-12.

22 Q. Zhu, E. Yildirim, X. Wang, X. Y. D. S. Soo, Y. Zheng, T. L. Tan, G. Wu, S.-W. Yang and J. Xu, Front. Chem., 2019, 7, 783.

23 T. A. Yemata, A. K. K. Kyaw, Y. Zheng, X. Wang, Q. Zhu, W. S. Chin and J. Xu, Polym. Int., 2020, 69, 84-92.

24 X. Wang, A. K. K. Kyaw, C. Yin, F. Wang, Q. Zhu, T. Tang, P. I. Yee and J. Xu, RSC Adv., 2018, 8, 18334-18340.

25 Y. Zheng, H. Zeng, Q. Zhu and J. Xu, J. Mater. Chem. C, 2018, 6, 8858-8873.

26 T. Park, C. Park, B. Kim, H. Shin and E. Kim, Energy Environ. Sci., 2013, 6, 788-792.

27 E. Yildirim, G. Wu, X. Yong, T. L. Tan, Q. Zhu, J. Xu, J. Ouyang, J.-S. Wang and S.-W. Yang, J. Mater. Chem. C, 2018, 6, 5122-5131.

28 Y. Xia and J. Ouyang, ACS Appl. Mater. Interfaces, 2012, 4, 4131-4140.

29 A. K. K. Kyaw, T. A. Yemata, X. Wang, S. L. Lim, W. S. Chin, K. Hippalgaonkar and J. Xu, Macromol. Mater. Eng., 2018, 303, 1700429.

30 T. A. Yemata, Q. Ye, H. Zhou, A. K. Kyaw, W. S. Chin and J. Xu, Hybrid Polymer Composite Materials, Elsevier, 2017, pp. 169-195.
31 H. Park, S. H. Lee, F. S. Kim, H. H. Choi, I. W. Cheong and J. H. Kim, J. Mater. Chem. A, 2014, 2, 6532-6539.

32 O. Bubnova, M. Berggren and X. Crispin, J. Am. Chem. Soc., 2012, 134, 16456-16459.

33 T.-C. Tsai, H.-C. Chang, C.-H. Chen and W.-T. Whang, Org. Electron., 2011, 12, 2159-2164.

34 S. H. Lee, H. Park, W. Son, H. H. Choi and J. H. Kim, J. Mater. Chem. A, 2014, 2, 13380-13387.

35 Z. Fan, P. Li, D. Du and J. Ouyang, Adv. Energy Mater., 2017, 7, 1602116.

36 Z. Fan, D. Du, X. Guan and J. Ouyang, Nano Energy, 2018, 51, 481-488.

37 N. Saxena, B. Pretzl, X. Lamprecht, L. Bießmann, D. Yang, N. Li, C. Bilko, S. Bernstorff and P. Muller-Buschbaum, ACS Appl. Mater. Interfaces, 2019, 11, 8060-8071.

38 T. A. Yemata, Y. Zheng, A. K. K. Kyaw, X. Wang, J. Song, W. S. Chin and J. Xu, RSC Adv., 2020, 10, 1786-1792.

39 T. A. Yemata, Y. Zheng, A. K. K. Kyaw, X. Wang, J. Song, W. S. Chin and J. Xu, Org. Electron., 2020, 81, 105682.

40 T. A. Yemata, Y. Zheng, A. K. K. Kyaw, X. Wang, J. Song, W. S. Chin and J. Xu, Front. Chem., 2020, 7, 870.

41 Y. H. Kim, C. Sachse, M. L. Machala, C. May, L. MüllerMeskamp and K. Leo, Adv. Funct. Mater., 2011, 21, 1076-1081.

42 A. M. Nardes, M. Kemerink, M. De Kok, E. Vinken, K. Maturova and R. Janssen, Org. Electron., 2008, 9, 727-734.

43 J. E. McCarthy, C. A. Hanley, L. J. Brennan, V. G. Lambertini and Y. K. Gun'ko, J. Mater. Chem. C, 2014, 2, 764-770.

44 A. Cho, S. Kim, S. Kim, W. Cho, C. Park, F. S. Kim and J. H. Kim, J. Polym. Sci., Part B: Polym. Phys., 2016, 54, 1530-1536.

45 D. Alemu, H.-Y. Wei, K.-C. Ho and C.-W. Chu, Energy Environ. Sci., 2012, 5, 9662-9671.

46 C. Yeon, S. J. Yun, J. Kim and J. W. Lim, Adv. Electron. Mater., 2015, 1, 1500121.

47 M. T. Z. Myint, M. Hada, H. Inoue, T. Marui, T. Nishikawa, Y. Nishina, S. Ichimura, M. Umeno, A. K. K. Kyaw and Y. Hayashi, RSC Adv., 2018, 8, 36563-36570.

48 D. A. Mengistie, M. A. Ibrahem, P. C. Wang and C. W. Chu, ACS Appl. Mater. Interfaces, 2014, 6, 2292-2299.

49 S. H. Lee, H. Park, S. Kim, W. Son, I. W. Cheong and J. H. Kim, J. Mater. Chem. A, 2014, 2, 7288-7294.

50 C. Yi, A. Wilhite, L. Zhang, R. Hu, S. S. C. Chuang, J. Zheng and X. Gong, ACS Appl. Mater. Interfaces, 2015, 7, 8984-8989.

51 J. Wang, K. Cai and S. Shen, Org. Electron., 2015, 17, 151-158.

52 X. Crispin, S. Marciniak, W. Osikowicz, G. Zotti, A. van der Gon, F. Louwet, M. Fahlman, L. Groenendaal, F. De Schryver and W. R. Salaneck, J. Polym. Sci., Part B: Polym. Phys., 2003, 41, 2561-2583.

53 Y. J. Xia and J. Y. Ouyang, Macromolecules, 2009, 42, 4141-4147.

54 M. Khan, S. Armes, C. Perruchot, H. Ouamara, M. Chehimi, S. Greaves and J. Watts, Langmuir, 2000, 16, 4171-4179.

55 T.-C. Chung, J. Kaufman, A. Heeger and F. Wudl, Phys. Rev. B: Condens. Matter Mater. Phys., 1984, 30, 702. 
56 S. Garreau, J. Duvail and G. Louarn, Synth. Met., 2001, 125, 325-329.

57 S. G. Im and K. K. Gleason, Macromolecules, 2007, 40, 6552-6556.

58 O. Bubnova, Z. U. Khan, A. Malti, S. Braun, M. Fahlman, M. Berggren and X. Crispin, Nat. Mater., 2011, 10, 429.

59 S. Garreau, G. Louarn, J. P. Buisson, G. Froyer and S. Lefrant, Macromolecules, 1999, 32, 6807-6812.

60 A. A. Farah, S. A. Rutledge, A. Schaarschmidt, R. Lai, J. P. Freedman and A. S. Helmy, J. Appl. Phys., 2012, 112, 113709.

61 Y. K. Han, M. Y. Chang, W. Y. Huang, H. Y. Pan, K. S. Ho, T. H. Hsieh and S. Y. Pan, J. Electrochem. Soc., 2011, 158, K88-K93.

62 J. Luo, D. Billep, T. Waechtler, T. Otto, M. Toader, O. Gordan, E. Sheremet, J. Martin, M. Hietschold, D. R. T. Zahn and T. Gessner, J. Mater. Chem. A, 2013, 1, 7576-7583.

63 M. Łapkowski and A. Proń, Synth. Met., 2000, 110, 79-83.

64 U. Lang, E. Müller, N. Naujoks and J. Dual, Adv. Funct. Mater., 2009, 19, 1215-1220.

65 J. Ouyang, Q. Xu, C.-W. Chu, Y. Yang, G. Li and J. Shinar, Polymer, 2004, 45, 8443-8450.

66 S.-I. Na, G. Wang, S.-S. Kim, T.-W. Kim, S.-H. Oh, B.-K. Yu, T. Lee and D.-Y. Kim, J. Mater. Chem., 2009, 19, 9045-9053. 67 J. Luo, D. Billep, T. Blaudeck, E. Sheremet, R. D. Rodriguez, D. R. Zahn, M. Toader, M. Hietschold, T. Otto and T. Gessner, J. Appl. Phys., 2014, 115, 054908.
68 J. Luo, D. Billep, T. Waechtler, T. Otto, M. Toader, O. Gordan, E. Sheremet, J. Martin, M. Hietschold and D. R. Zahn, J. Mater. Chem. A, 2013, 1, 7576-7583.

69 Z. Li, H. Sun, C. L. Hsiao, Y. Yao, Y. Xiao, M. Shahi, Y. Jin, A. Cruce, X. Liu, Y. Jiang, W. Meng, F. Qin, T. Ederth, S. Fabiano, W. M. Chen, X. Lu, J. Birch, J. W. Brill, Y. Zhou, X. Crspin and F. Zhang, Adv. Electron. Mater., 2018, 4, 1700496.

70 Z. Liu and N. Wang, J. Mater. Chem. C, 2018, 6, 9734.

71 N. Kim, S. Kee, S. H. Lee, B. H. Lee, Y. H. Kahng, Y. R. Jo, B. J. Kim and K. Lee, Adv. Mater., 2014, 26, 2268-2272.

72 S. Xu, M. Hong, X.-L. Shi, Y. Wang, L. Ge, Y. Bai, L. Wang, M. Dargusch, J. Zou and Z.-G. Chen, Chem. Mater., 2019, 31, 5238-5244.

73 N. Kim, B. H. Lee, D. Choi, G. Kim, H. Kim, J. R. Kim, J. Lee, Y. H. Kahng and K. Lee, Phys. Rev. Lett., 2012, 109, 106405. 74 J. Zhou, E. Q. Li, R. Li, X. Xu, I. A. Ventura, A. Moussawi, D. H. Anjum, M. N. Hedhili, D.-M. Smilgies, G. Lubineau and S. Thoroddsen, J. Mater. Chem. C, 2015, 3, 2528-2538.

75 T. Baba, N. Taketoshi and T. Yagi, Jpn. J. Appl. Phys., 2011, 50, 11RA01.

76 G.-H. Kim, L. Shao, K. Zhang and K. P. Pipe, Nat. Mater., 2013, 12, 719.

77 M. De Jong, L. Van Ijzendoorn and M. De Voigt, Appl. Phys. Lett., 2000, 77, 2255-2257.

78 K. Fehse, R. Meerheim, K. Walzer, K. Leo, W. Lövenich and A. Elschner, Appl. Phys. Lett., 2008, 93, 312. 